\title{
Editorial: Animal Genomes, Bodies and Tissue in Science and Society
}

The papers in this issue address the field of animal biotechnology and, particularly, animal genomics. Together they seek to understand the context and shaping of the science of animal genomics, reflect on connections between this science and the social position and cultural construction of animals and human-animal relationships, and explore current and future regulation and policy. Each paper emerged from a multi-disciplinary workshop convened by the ESRC Genomics Policy and Research Forum, University of Edinburgh to discuss animal genomics in April 2006. Some participants at the workshop, and some authors here, have long been interested in animal genomics and biotechnology, whilst others were asked to bring knowledge developed in other fields and with other case studies.

Underpinning the workshop, and followed through in this issue, was a recognition that genomics uses and studies a lot of animals, combined with concern that the otherwise flourishing social science of genomics has passed them over. The strength of concern is variously expressed. In my own editorial essay, I ask only that the animal becomes more conspicuous. Others call for something stronger: Donaldson, for example, argues that we need to rethink our idea of society or the social in such a way that the inclusion of nonhuman animals becomes obvious.

Whichever route is adopted, a handful of themes recur, including: whether animal genomic science is reductive; the extent to which it aligns with narratives of instrumentalism; the potential for animal genomics to render animals efficient sources or accumulators of capital; whether animal genomics functions as an instrument of biopower; and whether animal genomics alters both what we can and do know about an animal, and the animal that is known. On the way we visit the farmyard (Donaldson, Holloway \& Morris, Twine), the laboratory (Harvey, Hauskeller), and the sea (Costa \& Carvalho), although as Twine points out, genomics means that it is increasingly difficult to separate such spaces.

Although final judgement rests with the reader, I hope this issue makes a valuable contribution both to the social scientific and philosophical analysis of genomics, and to the growing field of animal studies. It also contains a new venture for the journal. The issue includes a broadly technical paper on DNA barcoding by molecular ecologists Filipe Costa and Gary Carvalho. Inclusion of a technical paper is new in itself, but moreover, this is followed by three short commissioned responses from John Dupré, a philosopher, Pete Hollingsworth, a conservation geneticist, and Petter Holm, a social scientist. Costa and Carvalho provide a final response and together these papers make for a very interesting discussion. Many aspects of animal genomics have been discussed before within social science journals, and sometimes from multiple perspectives in this way, but probably not DNA barcoding.

\section{Matthew Harvey}

ESRC Genomics Policy and Research Forum, University of Edinburgh, UK

Guest Editor 